\title{
La Théorie critique des médias de l'École de Francfort : une relecture
}

La théorie de " critique des médias " issue de l'École de Francfort est sans conteste l'une des plus importantes depuis le début du XX' siècle. C'est aussi l'une des plus discutées. Quels sont les contours et contenus de cette théorie critique ? Comment appréhender les objections qui lui ont été faites ? Répondant à ces questions, Olivier Voirol montre toute la richesse et la fécondité contemporaine de cette contribution théorique unique.

$\mathrm{P}$

armi les multiples "théories critiques " des médias qui ont vu le jour au siècle dernier, la Théorie critique de l'École de Francfort occupe incontestablement une place à part. Avec ses concepts de "perte de l'aura ", d' " unidimensionnalité " et surtout d'" industrie culturelle ", cette théorie a fait couler beaucoup d'encre et figure souvent en bonne place dans les manuels d'introduction aux théories des médias. Cependant, lorsqu'elle est évoquée, y compris pour souligner son importance, c'est souvent pour pointer ses limites et dénoncer le caractère suranné de sa critique. Force est donc de constater qu'elle n'est plus en vogue de nos jours. Qui saurait se réclamer du concept d'، industrie culturelle " aujourd'hui sans réveiller mille soupçons?

En France, tout particulièrement, où la théorie francfortoise des industries culturelles a été discutée dès le début des années $1960^{1}$, en partie grâce à Edgar Morin et au Centre d'études de communication de masse, elle a éveillé - et continue d'éveiller, jusqu'à aujourd'hui - une certaine méfiance, si ce n'est une critique virulente. S'inspirant des travaux de Theodor W. Adorno sur la culture de masse, Morin lui-même n'était pas avare en critiques puisqu'il voyait dans l'approche francfortoise une perspective trop homogène excluant toute part de créativité au sein des " industries culturelles ${ }^{2}$ ". À la suite de ces travaux, d'autres sociologues s'en sont pris à ces recherches sur la culture de masse en critiquant vertement une approche qui, selon eux, partait d'un concept problématique de "masse". Dans un texte qui a fait date, Pierre Bourdieu et Jean-Claude Passeron s'en prenaient ainsi à la "massmédiologie " abstraite, plongée

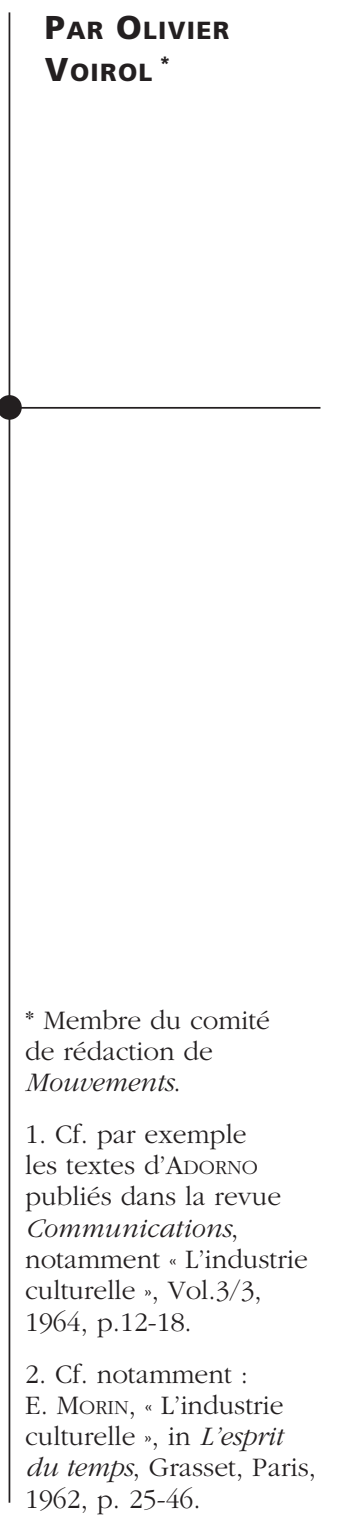

* Membre du comité de rédaction de Mouvements

1. Cf. par exemple les textes d'ADORNO publiés dans la revue Communications, culturelle ", Vol.3/3,

2. Cf. notamment : E. MORIN, "L'industrie du temps, Grasset, Paris, 1962, p. 25-46. 1964, p.12-18 
3. P. Bourdieu

\& J.-C. Passeron, "Sociologues des mythologies et mythologies de sociologues ", in Les Temps Modernes, 1963, n²4, p. 998-1021.

4. Cf. C. Lemieux, "Une critique sans raison? L'approche bourdieusienne des médias et ses limites ", B. LAHIRE (dir.), Le travail sociologique de Pierre Bourdieu,

La Découverte, Paris, 1999, p .205-229.

5. J'ai discuté le problème de cette posture : cf. "Idéologie : concept culturaliste et concept critique ", in Actuel Marx, $\mathrm{n}^{\circ} 43,2008$, p. $62-78$. dans un " discours prophétique (...) sur les nouveaux moyens de communication" et succombant à une "vulgate pathétique "3. Si cette critique était avant tout adressée à leurs collègues du Centre d'études de communication de masse, elle atteignait (et égratignait) aussi les tenants de la Théorie critique de l'école de Francfort.

Au cours de ces dernières années, la montée en puissance des théories de la réception et des perspectives insistant sur la réappropriation et le détournement des messages par leurs destinataires, l'approche francfortoise des médias a souvent fait office de repoussoir, incarnant, à la limite, tout ce qu'il ne faut pas faire en termes d'étude de la communication et des médias. L'intérêt actuel pour les Cultural studies, longtemps boudées en France, sert parfois de tremplin à une rengaine critique contre l'approche francfortoise des médias et de la culture. Quant aux tenants d'une approche dite "pragmatique " de la critique des médias, ils ont contribué à mettre indistinctement dans le même sac la "critique sans raison " de Bourdieu et de ses partisans ${ }^{4}$ avec celle de l'École de Francfort. S'il fallait résumer en quelques lignes les tenants et aboutissants de ces objections adressées à la critique francfortoise des médias, on pourrait dire qu'elles reposent essentiellement sur trois éléments.

Tout d'abord, selon une objection classique, la théorie francfortoise aurait exagéré le pouvoir des " industries culturelles " en concevant leurs destinataires comme dépourvus de capacité d'interprétation, de réappropriation, voire de résistance. Les énoncés et les images médiatiques seraient " reçus " par les destinataires de la même manière qu'ils ont été conçus du côté de la production - sans que n'intervienne un contexte d'action et d'interaction, un univers culturel propre à un groupe social, une classe sociale, un genre, etc. En cela, cette théorie aurait eu la naïveté de croire que les messages médiatiques se répercutent tels quels dans les expériences des individus, réduits à des sujets passifs et manipulés, sinon mystifiés, par les " industries culturelles ".

Puisque cette critique des médias présuppose, croit-on, des institutions médiatiques toutes puissantes et puisque les individus sont vus comme des sujets passifs dénués de toute compétence critique, la Théorie critique serait condamnée à adopter une position " de surplomb " par rapport à l'univers des pratiques sociales. Par le constat unilatéral d'une dépossession des sujets, ces théoriciens adopteraient une critique "externe " au point de vue des acteurs sociaux : ces derniers seraient incapables de "voir " par eux-mêmes les conditions d'oppression dans lesquelles ils se trouvent - ce qui exigerait alors une distance théorique capable de " dévoiler " cette soumission 5 . Pire, selon cette objection, les tenants de la Théorie critique se placeraient non seulement dans cette posture d'extériorité, ils se défileraient aussi devant la nécessité de clarifier le point de vue à partir duquel s'énonce cette critique.

Enfin, ce qui est le plus souvent reproché à cette Théorie critique des médias, c'est son caractère "spéculatif ", éloigné de la recherche empirique et de la concrétude de l'enquête sur les pratiques culturelles et médiatiques. Selon cette même critique, seul un modèle philosophique et 
abstrait permettrait de développer cette critique des médias sans ancrage dans les conditions effectives de réception des biens culturels et médiatiques produits par les industries culturelles, et sans procéder à une analyse sérieuse des contenus de ces dernières.

D'autres objections pourraient être évoquées si nous en avions la place. Toutefois, il ne s'agit, ici, que de mentionner les grands axes de ces objections afin de les mettre en discussion. Force est de constater que ces critiques font le plus souvent une lecture superficielle, limitée à la lecture de quelques textes épars, sans replacer la critique francfortoise des médias dans son cadre théorique général. Ce modèle de la critique des médias apparaît alors sous une forme particulièrement naïve qui occulte sa profondeur théorique et sa teneur conceptuelle. Par ailleurs, cette lecture lacunaire n'est pas étrangère au rejet massif dont la théorie francfortoise des médias est victime en France et explique en partie son insignifiance dans la recherche actuelle sur les médias et la communication sociale en langue française.

Dans les pages qui viennent, j'aimerais revenir sur cette critique des médias en proposant une lecture différente, notamment en la réinscrivant dans le projet d'ensemble de la Théorie critique, en particulier la version proposée par Adorno. En effet, cette tradition intellectuelle défend à mes yeux une conception originale de la critique de la culture et des médias, qu'on ne peut assimiler aux autres approches critiques des médias (par ex. Bourdieu, Chomsky, Foucault, Gramsci, etc.) sans omettre ses spécificités. À cela s'ajoute le fait que cette critique des médias - contrairement à ce qu'inspire une lecture pressée du fameux chapitre de la Dialectique de la raison consacré à l'" industrie culturelle " ${ }^{6}$ - n'est pas donnée "clé en main ", à l'image d'un programme théorique cohérent censé se déchiffrer aisément dans ses formulations générales. Au contraire, ce modèle ne se révèle dans sa finesse que lorsqu'on le réinscrit dans le projet d'ensemble de la théorie de la société développée par la Théorie critique.

À partir de la lecture de ce fameux chapitre de la Dialectique de la raison, de Adorno et Horkheimer, on peut retenir de cette critique des médias les éléments suivants. (a) Dans le type de capitalisme qui se développe dès le début du $\mathrm{XX}^{\mathrm{e}}$ siècle, la valorisation marchande ne se limite plus à l'échange de la force de travail au sein de la sphère économique, comme aux temps de Marx, elle s'étend désormais aux domaines de la "culture " (art, médias, connaissance) jusque-là épargnés, en partie du moins, par cette dernière. (b) Cette soumission à la valorisation marchande n'est pas sans conséquences sur le contenu des biens culturels : ce sont leurs "qualités internes " qui se dégradent et s'appauvrissent dès lors qu'ils s'homogénéisent et se standardisent pour devenir des " pro-
6. T.W. ADORNO \& M. HORKHEIMER, "La production industrielle des biens culturels ", in Dialectique de la raison, Gallimard, Paris, 1974 (1947). 
7. Je me concentre ici exclusivement sur la théorie de l'industrie culturelle d'ADORNO \& HorkHEIMER, qu'il conviendrait de distinguer des apports de Benjamin, Kracauer ET MarCuse.

8. Cf. M. Horkheimer, Théorie critique et théorie traditionnelle, Gallimard, Paris, 1974.

9. Après l'abandon de modèle de la praxis sociale dans les années 1940, Adorno situera ces potentiels dans la sphère esthétique, seul " lieu " encore susceptible d'incarner les principes d'une culture émancipée. duits comme les autres "sur le marché capitaliste. C'est "à la chaîne " que se produisent des films, des émissions de radio et de télévision, des morceaux de musique à succès, à l'instar de n'importe quel autre produit de consommation de masse. (c) Soumise à la valeur d'échange, la culture est mobilisée à des fins instrumentales : de "finalité sans fin " (Kant), elle devient "opérationnelle ", ayant vocation à " servir " certaines finalités. (d) Les médias modernes (radio, télévision, cinéma) ne remettent pas en cause le monde " tel qu'il est " mais le reconduisent dans un conformisme de tous les instants-alors même qu'ils se nourrissent d'une force d'attraction culturelle dont les ressorts reposent précisément sur la distance face à "ce qui est ". (e) Ces mêmes médias produisent des images et des discours qui s'imprègnent dans la conscience d'" individus faibles " et incapables de se les réapproprier de manière critique; ils ne sont pas - ou plus - à même de résister efficacement aux produits des mass media.

\section{- La critique des médias}

Telle peut être, résumée en quelques "thèses ", la conception de l'industrie culturelle qui est au cour de la critique des médias dans la Théorie critique ${ }^{7}$. Dès lors que ces bases sont clarifiées, il est possible de décrire de manière plus complexe l'approche francfortoise des médias, pour montrer que les objections auxquelles elle a été soumise n'atteignent que très partiellement leur cible. Cela revient, par conséquent, à esquisser les axes d'une autre lecture de cette critique des médias. Pour ce faire, il convient de prendre en considération trois aspects majeurs de la Théorie critique, qui échappent à une lecture superficielle : premièrement, le fait, on l'a dit, que la critique des médias s'inscrit dans une critique générale de la société et qu'elle n'est pas indépendante de cette dernière ; deuxièmement, le fait que l'accent mis sur la dépossession des sujets sociaux n'est pas basé sur une conception " externe " mais " interne " de la critique ; troisièmement, loin d'être rivée à une conception spéculative, cette critique des médias part d'analyses concrètes menées sur les produits de la culture de masse. Je reviendrai dans un instant sur ces trois points.

Avant cela, j'aimerais insister sur la forme particulière du schéma théorique d'ensemble dans lequel Adorno et Horkheimer placent leur critique des médias. Celui-ci part explicitement du point de vue de l'“ intérêt à l'émancipation ": Max Horkheimer définit la Théorie critique comme une posture théorique qui, loin de se situer en extériorité par rapport aux pratiques sociales, prend ancrage dans ces dernières ${ }^{8}$. Selon lui, une théorie menant une critique des processus sociaux ne saurait se satisfaire d'une posture externe consistant à postuler de manière idéale et extérieure le monde "tel qu'il "devrait être" ". Au contraire, elle doit partir des pratiques effectives et des potentialités incarnées en elles, dont on peut rendre compte sur le plan théorique du caractère émancipateur. En deux mots, dans le modèle fondateur de la Théorie critique (années 1930), ces potentiels émancipateurs relèvent des forces de la raison, incarnées dans le processus historique et les pratiques sociales qui le portent ${ }^{9}$. La raison 


\section{La Théorie critique des médias de l'École de Francfort : une relecture}

se dresse contre l'emprise des mythes, de la nature, des formes traditionnelles d'oppression et invite, à l'inverse de l'idéologie, au contrôle conscient de la collectivité sur elle-même.

C'est à cet idéal de la raison que se rattache le projet moderne de la culture émancipée. Selon Adorno et Horkheimer, l'idée de culture est en effet, depuis le siècle des Lumières, liée au principe de la raison émancipatrice. Une des conquêtes de la société moderne, au sortir du Moyen Âge, est d'avoir permis une autonomisation de la culture et de l'art, arrachés aux puissances du religieux et au pouvoir politique. Sous la forme de pratiques artistiques, de modes d'éducation, de produits culturels émancipés des tutelles antérieures, tout un domaine que l'on peut comprendre sous le terme de "culture " devient un des lieux effectifs d'expression de la raison émancipatrice.

Cette culture attachée à la raison est vue de deux manières : d'une part, comme une pratique immanente, située dans les pratiques sensibles des acteurs - et notamment dans la praxis sociale et dans l'activité esthétique ; d'autre part, comme un idéal transcendant, une promesse inscrite " contre ce qui est " et qui contient toujours plus que ce que la pratique peut réaliser ${ }^{10}$. C'est cette articulation entre immanence et transcendance qui permettra, d'un côté, de critiquer, au nom de la pratique, la conception bourgeoise de la culture et de l'art comme sphère culturelle idéale coupée du monde pratique ${ }^{11}$ et, d'autre part, de critiquer au nom de la "promesse de bonheur " non réalisée toute réduction de la culture à la pratique et au monde " tel qu'il est ". C'est dans cette articulation dialectique que s'inscrit la critique des médias.

À l'arrière-plan des analyses des membres de l'école de Francfort (de Marcuse à Adorno, en passant par Horkheimer, Kracauer et Löwenthal) se trouvait sans conteste cette conception de la culture articulée à la Raison et à l'émancipation. C'est à l'aune de ce référent qu'ils ont observé la montée des médias et de la culture de masse - apparition de la presse magazine, popularisation du cinéma et de la radio, émergence de la télévision, etc. Leurs observations sur les médias et la culture de leur temps, autant que sur les processus politiques, sont fondamentalement gouvernées par la conviction dans les potentiels émancipateurs de la culture.

Cette conviction dans les potentiels d'une culture émancipatrice a donc gouverné leur critique des médias et, surtout, a guidé l'élaboration du concept d'" industrie culturelle ". Ce terme à présent banalisé était un oxymoron à cette époque : quoi de plus antinomique que la culture et l'industrie ? Autrement dit, le constat revient à dire que la culture est soumise aux modes d'organisation et aux processus instrumentaux caractéristiques de la sphère économique. En suivant les analyses de Georg Lukács sur la réification, les francfortois sont convaincus que le propre de la culture dans la phase monopolistique que connait le capitalisme dans le premier tiers du $\mathrm{XX}^{\mathrm{e}}$ siècle, est d'être envahi, au même titre que tous les autres domaines de la vie sociale, par la valorisation marchande et la rationalisation industrielle. Ils ont vu la progression de l'échange marchand dans le domaine de la culture et des médias comme une remise en cause,
10. T. W. AdORNO, "Critique de la culture et société ", in Prismes, Payot, Paris, 2003, p. 7-23.

11. Cf. H. Marcuse, "Sur le caractère affirmatif de la culture " in Culture et société, Minuit, Paris, 1970. 
12. S. Kracauer a été le premier à formuler cette idée dans sont texte, "L'ornement de la masse ", in L'Ornement de la masse, ed. par O. AGARD, La Découverte, Paris, 2008 , p. 60-71.

13. T. Adorno, Le fétichisme en musique et la régression de l'écoute, Allia, Paris, 2008 (1938).

14. C'est là un des points de discorde entre ADORNO et LAZARSFELD dans le Princeton Radio Project, qui entraînera le départ d'Adorno de ce dernier.

15. Je me permets ici de renvoyer à mon texte : "Médiations et Théorie critique ", in Réseaux, $n^{\circ} 148-149,2008 / 2-3$, p. 47-78. sinon une destruction systématique, du processus historique d'émancipation par la raison et la culture moderne. Selon eux, si le marché, dirigé contre les mécanismes traditionnels de dépendance et de tutelle liés à des rangs sociaux, avait ouvert, à la fin du XVIII ${ }^{\mathrm{e}}$ siècle, un espace culturel libéré des tutelles du religieux et du pouvoir, il s'est entre-temps transformé en pure mystification. Le rapport marchand est un principe d'oppression qui masque la profondeur des rapports sociaux et réinstalle les individus dans des relations obscures ${ }^{12}$. Parce qu'il masque le fondement du rapport social sur lequel il s'appuie, le rapport marchand contribue à la réémergence d'une relation mystifiante au réel et d'un " rapport social d'aveuglement "(Adorno).

La culture moderne, qui porte en elle-même une promesse d'émancipation et de liberté extérieure à la valorisation capitaliste et à tout rapport instrumental, devient donc le socle de processus marchands et industriels. C'est à ce type de critique que sont soumis les médias : à des fins commerciales, ils font mine de réaliser un projet culturel qu'ils contribuent dans les faits à miner. On produit des séries télévisées comme on fabrique des savonnettes ou des voitures, selon une gestion industrielle gouvernée par la rationalité instrumentale. Selon Adorno et Horkheimer, on assiste, notamment par le biais de l'industrie culturelle, à un détournement de la culture et un retournement du processus d'émancipation en son contraire. Ce processus atteint la culture non pas de manière superficielle mais dans son contenu même. Adorno le disait dans un texte précoce consacré à la "régression de l'écoute ${ }^{13}$ ": le fait de soumettre la musique à la valorisation marchande a une portée sur les "qualités internes " de cette dernière et sur le type d'expérience esthétique permise aux auditeurs. La musique marchande s'offre facilement à l'écoute pour être plus vendable, elle fait dans l'effet et la figure de style - notamment sous la forme d'arrangements - pour compenser en surface ce qu'elle ne donne plus en profondeur, en termes d'expérience musicale.

Adorno a accordé une attention particulière aux qualités internes des œuvres musicales et aux produits médiatiques dont l'étude ne doit, à ses yeux, être découplée de celle des processus sociaux. Il refuse en effet de prendre comme unique point de référence l'expérience de réception des destinataires de produits médiatiques sans relier cette dernière aux contenus culturels qui sont au fondement de cette expérience ${ }^{14}$. Et c'est notamment parce qu'Adorno prend en considération la "relation " entre ces différentes instances qu'il ne veut occulter la valorisation capitaliste appliquée à la culture. Son approche s'appuie fondamentalement sur le concept de "médiation " pour rendre compte de la manière dont processus socioéconomiques et contenus culturels s'interpénètrent de manière complexe, selon des modalités qu'il est néanmoins impossible de définir a priori sans un examen rigoureux des contenus culturels ${ }^{15}$.

Cette approche de la relation entre les qualités internes des biens culturels, l'expérience culturelle des sujets et les processus socio-économiques, s'ajoute également un ensemble de considérations sociologiques issues de la recherche empirique, qui contribuent également à saisir la 
teneur de cette expérience possible. Adorno est convaincu que cette expérience culturelle, à l'instar des contenus culturels, doit être ramenée à l'expérience sociale des individus dans les conditions du "capitalisme monopolistique ". Un des constats majeurs dressé par les Francfortois est que l'expérience de la réception des contenus médiatiques ne saurait être examinée en elle-même et doit être reliée aux processus de formation des individus. Or, selon le constat dressé par les membres de cette "École " dès le début des années 1930, à la suite d'une grande enquête consacrée aux mutations de la famille et de la formation de la personnalité, les sujets modernes sont de plus en plus dans l'impossibilité de développer un soi "fort " et une autonomie personnelle. Inspirée par la psychanalyse, cette enquête montre que ces derniers, inscrits dans un processus de socialisation et de construction de soi (disparition de la figure du père indépendant

Un des constats majeurs dressé par les Francfortois est que l'expérience de la réception des contenus médiatiques ne saurait être examinée en elle-même et doit être reliée aux processus de formation des individus.

dans une famille éclatée sou-

mise au capitalisme de la grande

industrie) qui leur ôte toute possibilité de se confronter à des instances d'autorité immédiate, tendent à se soumettre à des instances d'autorité secondaires (partis politiques, institutions, État, leaders politiques et religieux, etc.).

Là encore, on comprend le lien entre ce diagnostic et la critique des médias : loin de prétendre que les médias modernes sont tout-puissants " en eux-mêmes ", cette approche vise à comprendre les transformations sur le plan des rapports sociaux, de l'économie et de la culture qui contribuent à l'apparition de sujets disposés à s'en remettre sans résistance à des institutions puissantes dont font partie les médias. Autrement dit, les considérations sur la réception proprement dite sont réinscrites dans une série de processus qui ne concernent pas directement les médias mais qui informent le type de rapport que les sujets sociaux entretiennent avec eux. Par conséquent, il s'agit moins, pour la Théorie critique, de pointer la toute-puissance des médias que d'identifier les processus qui font des sujets modernes des "individus faibles ", n'ayant d'autre recours que de s'en remettre à des institutions dont les orientations leur échappent.

\section{- Renouveler la critique des médias}

À partir de ces remarques, il est possible de revenir sur les objections évoquées plus haut, adressées à l'approche francfortoise des médias, en montrant comment elle est largement en mesure de leur donner réponse.

Premièrement, face aux objections postulant l'absence d'activités interprétatives des sujets sociaux du côté de la "réception ", on a en réalité affaire à un modèle complexe qui inscrit la critique des médias non seulement dans un examen général de la société - en particulier des processus 
de socialisation et de la formation des sujets sociaux -, mais qui appréhende également les " médiations " entre contenus culturels, expériences des sujets sociaux et processus socio-économiques. Fidèle au projet de la Théorie critique qui entend saisir les transformations historiques et les processus sociaux en les appréhendant comme des modes de manifestation de l'ensemble sociétal (grâce à une démarche interdisciplinaire), ce modèle critique insère l'étude des médias dans un diagnostic de la situation historique et la relie à une analyse impliquant l'ensemble des institutions sociales - notamment l'évolution du capitalisme et le devenir de l'individu. On pourrait ajouter à cela que cette étude ne peut être formulée de manière spéculative et qu'elle implique le recours à la recherche empirique. Cette critique des médias se caractérise donc par sa capacité de réinscrire les processus culturels et les institutions médiatiques dans un ensemble sociétal qui les dépasse largement. Ce qui a pour conséquence, d'une part, que l'on ne peut critiquer les évolutions que prennent les médias modernes en les détachant du contexte socio-économique et politique dans lesquels ils sont inscrits et, d'autre part, que le rapport que les sujets sociaux entretiennent avec les contenus culturels doit être appréhendé dans ce tout.

Deuxièmement, au vu de ce qui a été dit plus haut, on comprend que les objections dénonçant la critique francfortoise des médias comme critique "de surplomb", extérieure à l'univers des pratiques sociales, au profit d'une posture d'extériorité, et d'une critique se défilant devant la clarification de son point de vue, n'est pas tenable. Les penseurs de Francfort défendent au contraire une conception selon laquelle il n'est possible de poser un constat critique sur une réalité sociale donnée que si l'on dispose d'un point de référence pré-théorique dans les pratiques sociales elles-mêmes. On est loin de l'idée selon laquelle ces penseurs se contenteraient de dénoncer de manière spéculative l'aliénation

\section{Cette critique des médias se caractérise par sa capacité de réinscrire les processus culturels et les institutions médiatiques dans un ensemble sociétal qui les dépasse largement.}

et la perte d'individualité des êtres humains dans le monde moderne sans jamais fournir le point de vue interne aux pratiques sociales à partir duquel cette critique s'énonce. Pour mener à bien leur critique de la culture médiatique, Adorno et Horkheimer disposaient bien d'un référent critique inscrit dans le principe moderne de la Raison et de la culture émancipatrice. S'ils se sont livrés à la critique des médias modernes, c'est parce que ces derniers ne participent plus selon eux de cet idéal et tendent à le pervertir, pour enfermer les êtres humains dans une irrationalité mythologique et leur ôter toute capacité d'agir de manière autonome.

Enfin, la troisième objection, celle qui s'en prend au caractère spéculatif de la critique francfortoise des médias, ne tient pas tant cette dernière est 
ancrée dans une analyse "interne " des qualités cultuelles. Cette critique des médias part en effet d'analyses concrètes sur les médias et la culture de masse. L'insistance sur les relations entre les processus socio-économiques et les contenus culturels pourrait certes laisser croire que ce modèle de critique des médias se contente alors d'en déduire mécaniquement des contenus culturels produits par ces derniers. On trouve ce type de causalité dans certaines approches relevant de l'économie politique de la communication, qui tendent à tirer un lien de causalité direct entre le type de structure économique et sociale dans lesquelles sont produits les discours médiatiques et la structure sémantique de ces derniers. Une des subtilités supplémentaires de la critique francfortoise des médias est précisément de se distinguer de ce modèle mécaniste postulant une relation immédiate entre contenus culturels et processus socio-économiques, modèle qu'elle considère comme simpliste ${ }^{16}$. Elle refuse un lien de causalité directe au profit d'un examen interne méticuleux des contenus culturels, des messages, de la musique commerciale, des propositions linguistiques, etc.

\section{- Conclusion}

La critique des médias menée par la Théorie critique se distingue des autres théories critiques des médias en vertu de l'articulation originale qu'elle propose entre les dimensions dégagées ici. C'est parce que la critique des médias n'est pas découplée de la critique de la société que cette approche a souvent fait l'objet de mécompréhensions et d'objections faciles - parce que les textes de critique des médias sont lus comme des contributions particulières à un domaine spécifique déconnecté du diagnostic d'ensemble, laissant croire à une certaine naïveté dans le postulat d'une toute puissance des médias de masse.

Si la complexité de cette critique des médias en fait une contribution unique aux théories critiques des médias au $\mathrm{XX}^{\mathrm{e}}$ siècle, ce sont ces mêmes qualités qui rendent difficile sa réactualisation. Dès lors que cette critique des médias s'inscrit à la fois dans une conception de la culture comme émancipation et dans une compréhension des principales mutations socio-économiques de l'époque (modèle de capitalisme, formation du sujet, famille, etc.), elle implique un cadre théorique particulièrement exigeant et, du coup, difficile à réactualiser. On peut, à juste titre, discuter longtemps de la pertinence d'adapter cette critique aux formes contemporaines de la culture et de la communication médiatiques ; on peut aussi discuter, au vu des mutations récentes de la culture et des médias de communication, de la pertinence actuelle du concept d'" industrie culturelle ", développé à un moment (au milieu du $\mathrm{XX}^{\mathrm{e}}$ siècle) où les médias n'étaient pas ce qu'ils sont aujourd'hui. Si le contexte historique et les médias qui nous environnent sont indéniablement différents de nos jours, ce modèle critique ne saurait être repris sans modifications. Cependant, à mon sens, ses principales articulations conceptuelles gardent leur actualité. Pour être ajustée au monde contemporain, cette critique des médias doit néanmoins répondre à une série de défis, notamment celui de se situer par rapport à la "révolution numérique " de ce début de $\mathrm{XXI}^{\mathrm{e}}$ siècle.
16. Cf. entre autres, Theodor W. Adorno, Introduction à la sociologie de la musique, Ed. Contrechamps, Genève, 1994. 
17. C'est le projet du groupe de recherche sur la culture et les médias que je coordonne à l'Institut für Sozialforschung de Francfort, qui porte en particulier sur les mutations actuelles de la télévision et de la culture numérique (http://www.ifs.unifrankfurt.de).
Pour finir, je me contenterai d'évoquer quelques enjeux de cette réactualisation. Tout d'abord, elle implique une approche qui décloisonne l'étude des processus de communication en réinscrivant ces derniers dans l'ensemble des processus sociaux (formation des sujets contemporains, modèle de capitalisme actuel, rapports entre culture et économie, etc.) Une telle perspective doit être à même de comprendre les processus sociaux qui contribuent à l'émergence de " sujets critiques et autonomes " dans leurs pratiques d'interprétation des médias et de la culture. Ensuite, elle suppose de disposer d'un "référent pratique " offrant un point de repère normatif et préthéorique permettant, d'une part, de concevoir des formes émancipatrices de créations culturelles et d'autre part, de mener une critique systématique de ce qui contribue à les oblitérer, les empêcher ou les détruire. Cela implique une évaluation de la portée contemporaine du concept classique (émancipatoire) de culture - qui donne sur bien des aspects une impression d'essoufflement. Enfin, il convient de disposer d'une méthodologie permettant une " analyse interne " des produits médiatiques et des biens culturels, capable en même temps de relier cette analyse à des processus " externes ". On comprend aisément l'immense défi et la difficulté que représente une actualisation de cette critique des médias pour les recherches actuelles s'inspirant de la Théorie critique $^{17}$. Un défi qui mérite néanmoins d'être relevé. 